\title{
Didáctica de la lectura basada en metacognición
}

\section{Reading didactics based on metacognition}

\author{
Álvaro William Santiago Galvis \\ Myriam Cecilia Castillo Perilla \\ Dora Luz Morales Casteblanco ${ }^{1}$
}

\section{Resumen}

Este texto presenta parte de los resultados que se obtuvieron en la realización del proyecto de investigación: “Diseño, elaboración y validación de una propuesta para la didáctica de la lectura fundamentada en metacognición para la educación básica", DLE-031-06. Para ello, se da cuenta de los objetivos propuestos, la metodología y los instrumentos que se utilizaron para el desarrollo del proyecto y los resultados que se obtuvieron de la aplicación del cuestionario metacognitivo, el cual formó parte del proceso de intervención didáctica.

\section{Palabras clave}

Lectura, comprensión, metacognición, pedagogía, didáctica, procesos cognitivos, estrategias metacognitivas.

\section{Abstract}

This article presents some of the results that were obtained in the development of the research project "Design, elaboration and validation of a didactics proposal of reading based on metacognition for elementary education", DLE-031-06. For this reason, the report includes the proposed objectives, the methodology and the instruments that were implemented for the development of the project; the results that were collected in the application of the metacognitive survey, which was a very important component during the second phase of the project.

\section{Keywords}

Reading, comprehension, metacognition, pedagogy, didactics, cognitive processes, metacognitive strategies.

Artículo recibido el 28 de julio y aprobado el 16 de octubre de 2009.

1 Profesores del Departamento de Lenguas de la Universidad Pedagógica Nacional, miembros del Grupo de Investigación en Pedagogía, Lenguaje y Comunicación, Gipelec. Correo electrónico: asantiago@pedagogica.edu.co 


\section{Presentación}

El manejo adecuado del código escrito, en lo que respecta a su comprensión y producción -los procesos básicos de la actividad lingüística del ser humano-,constituye uno de los aprendizajes más valiosos que puede ofrecer la escuela al individuo, dado que por medio del dominio de este sistema puede tener acceso a -y formar parte de- la cultura alfabetizada en la que se encuentra inmerso. Si bien a la escuela se le ha asignado, entre otros aspectos, la misión de alfabetizar a los individuos, esto no se ha conseguido del todo y, como consecuencia de ello, ha surgido la noción de fracaso escolar que, según Ferreiro (2002), está asociada a un problema en la enseñanza que afecta, desde luego, el proceso de aprendizaje.

La realidad académica de los estudiantes, en lo que a lectura y escritura se refiere, lleva a plantear que muchos de los problemas en su desempeño escolar, al igual que en el profesional y laboral después, están relacionados con deficiencias en su formación lectora y escritora. En este marco, se hace evidente la necesidad de plantear proyectos y programas de investigación que apunten a proponer soluciones y, así, replantear las maneras de abordar la lectura, y en consecuencia el código escrito, en la escuela, y especialmente, con el fin de empezar a formar lectores o ciudadanos de la cultura alfabética; esto es, citando a Lerner (2001), individuos que han integrado la lectura como parte de sus actividades cotidianas, que saben seleccionar los textos pertinentes para solucionar problemas, que comprenden, interpretan y disfrutan los textos con los que interactúan.

Con el fin de afrontar el problema antes descrito, el proyecto "Diseño, elaboración y validación de una propuesta para la didáctica de la lectura fundamentada en metacognición para la educación básica", DLE-031-06, asumió como hipótesis de trabajo que los estudiantes con quienes se adelanta un trabajo en metacognición cualifican sus procesos de lectura; hecho que les permite, por un lado, conocer y reconocer sus dificultades al leer $y$, por el otro, encontrar y proponer soluciones para resolverlas. De esta forma, se propone que el trabajo en lectura con una base metacognitiva posibilita el mejoramiento de este proceso, puesto que los individuos conocen el objetivo de la tarea lectora, adelantan autoobservación de dicha tarea y llevan a cabo la autorregulación de los procedimientos implicados en ésta.

\section{Objetivo}

Para el proyecto DLE-031-06 se definió como propósito fundamental: diseñar, elaborar y poner a prueba un programa de intervención para la enseñanzaaprendizaje de la lectura, teniendo como sustento teórico la metacognición. Con el fin de cumplir con este propósito, el trabajo se dividió en dos grandes fases. En la primera, se estableció como finalidad el diseño y la elaboración de la propuesta didáctica ${ }^{2}$ (2006), organizada en unidades didácticas, las cuales se delimitaron a partir de la definición de los procesos cognitivos a saber: centrar la atención, analizar, organizar y elaborar ${ }^{3}$. Cabe señalar que se tomó la unidad didáctica como el elemento de trabajo básico para la formulación de la propuesta; este elemento se asumió como una posibilidad de configuración didáctica, con base en la cual se elaboraron las propuestas de planificación didáctica para los procesos cognitivos antes señalados, y específicamente con su respectivo componente metacognitivo ${ }^{4}$.

2 Para dar cuenta de este objetivo: 1. Se adelantó una revisión bibliográfica así como una discusión sobre didáctica de la lengua materna y didáctica de la lectura, con el fin de ver la relación entre estos conceptos y definir el enfoque didáctico que sustentaría la propuesta. 2. Se llevó a cabo la recuperación, sistematización, socialización y análisis de la práctica de la docente que tuvo bajo su orientación la intervención en la institución educativa; este trabajo se constituyó en un ejercicio de autorreflexión y autoanálisis, por parte de la docente, sobre la manera como ha abordado, a lo largo de su trayectoria profesional, la enseñanza de la lectura en la educación básica primaria; aspecto que permitió confrontar dicha experiencia con la teoría revisada en relación con la enseñanza de la lectura. 3. Se hizo una revisión bibliográfica en torno a la noción de metacognición; asimismo, se adelantó un trabajo de indagación bibliográfica en relación con el tema de la enseñanza de estrategias, lo que llevó a clarificar y precisar las nociones de estrategia cognitiva y estrategia metacognitiva, la relación entre ellas y el papel de éstas en la lectura y el proceso de enseñanza-aprendizaje de la misma. 4) Finalmente, se elaboró la propuesta didáctica para la enseñanzaaprendizaje de la lectura con una base metacognitiva.

3 Para el grado cuarto se establecieron tres unidades de trabajo: centrar la atención, analizar y organizar; y para el grado quinto, las unidades planteadas fueron: centrar la atención, analizar, organizar y elaborar.

4 Para tener una aproximación al marco conceptual que sustenta el trabajo en cuestión se puede consultar el trabajo: Santiago, Castillo y Morales, 2007 
En la segunda fase, se ejecutó dicha propuesta por medio de una intervención didáctica en la Institución Educativa Distrital Colegio Femenino Lorencita Villegas de Santos-Obrero Unión Social, actividad que se desarrolló durante el año lectivo 2007. Así, para la segunda fase, el objetivo del trabajo se orientó hacia la validación de la propuesta didáctica por medio de una intervención guiada, intervención que, se desarrolló en los grados 402 y 501 de la institución antes señalada, bajo la coordinación de la docente Dora Luz Morales Castiblanco, profesora del área de lengua castellana en la institución e integrante del equipo investigador 5 .

\section{Metodología}

El trabajo se adelantó desde la perspectiva del modelo de la investigación educativa, la cual forma parte de las variantes que en educación ha tomado la investigación-acción. Esta orientación centra su interés en las problemáticas que el docente experimenta en su labor cotidiana, de tal forma que en este modelo, se pretende que el educador se convierta, a la vez, en observador e investigador de su actividad pedagógica, cualificando así, su formación profesional y su práctica educativa. De acuerdo con Restrepo, la investigación-acción pedagógica: "se centra en los microprocesos de clase, en el desarrollo del currículo como objeto primordial" (Restrepo et ál., 2004, p. 24). Así, esta orientación metodológica se torna adecuada, dado que su objetivo está dirigido

5 La intervención supuso la realización de estas actividades: 1. Aplicación de un cuestionario metacognitivo con el fin de identificar qué conocimientos metacognitivos poseen las estudiantes con quienes se trabajaría durante el período 2007, así como el uso que hacen de éstos al momento de adelantar una tarea lectora. 2. Administración de una prueba de comprensión lectora para indagar sobre el grado de comprensión de las estudiantes, al iniciar y al finalizar la intervención. 3. Desarrollo de las unidades didácticas para trabajar los procesos centrar la atención, analizar y organizar, para el grado cuarto, y centrar la atención, analizar, organizar y elaborar, para el grado quinto. Actividad ésta que supuso la utilización de estrategias didácticas como el modelado, la práctica guiada, el trabajo cooperativo y la práctica autónoma, así como la utilización de guías, talleres y ejercicios de lectura. 4. Evaluación del trabajo de intervención y de los resultados que se obtuvieron de éste, a partir de los datos que se recopilaron a lo largo del desarrollo del proyecto, recopilación que se hizo por medio de los cuestionarios metacognitivos de entrada y salida, las pruebas de comprensión lectora de entrada y salida, los talleres y ejercicios que se adelantaron durante la intervención didáctica y los diarios de campo. a las problemáticas que se dan en la labor cotidiana de los docentes en las aulas, cómo abordarlas y cómo formular posibles soluciones a partir de propuestas de trabajo que transformen las prácticas, en pro de una mejor formación para los discentes, en este caso, en lo que a la lectura respecta, es el objetivo central de esta metodología.

Esta propuesta metodológica implica la reflexión sobre áreas problemáticas: por un lado, la planificación y ejecución de acciones para abordar dicha situación y por otro, la evaluación del proceso y los resultados de la intervención como tal (cfr. Restrepo et ál., 2004). Según ésto, en un primer momento del trabajo se adelantó una indagación en torno a la lectura y su didáctica y el papel que puede desempeñar la metacognición en este proceso; luego, a partir de este análisis, se formuló una propuesta de intervención didáctica, en la que se contemplan aspectos cognitivos y metacognitivos asociados al proceso lector, propuesta que se llevó al aula de clase y se puso en ejecución; finalmente, se evaluó la incidencia del trabajo realizado en la mejora del proceso lector del grupo de estudiantes. Según lo anterior, para el desarrollo del proyecto se establecieron las siguientes etapas:

a) Reflexión sobre el área problemática, que en este caso es el proceso de enseñanzaaprendizaje de la lectura.

b) Formulación de la propuesta para la didáctica de la lectura, basada en metacognición, teniendo como referente algunos de los procesos cognitivos implicados en el proceso lector y las estrategias metacognitivas asociadas a éste, las cuales apuntan a planificar, supervisar y evaluar el desarrollo de la tarea cognitiva.

c) Ejecución de la propuesta didáctica en el aula de clase. Para este caso, en los grados cuarto y quinto de educación básica primaria, de la Institución Educativa Distrital Lorencita Villegas de Santos-Obrero Unión Social.

d) Evaluación de la ejecución de la propuesta didáctica y del proyecto en general. 
Según lo expuesto, la metodología general del proyecto estuvo conformada por tres fases, que incluyeron cuatro subfases, las cuales, a su vez, estuvieron conformadas por unos momentos que implicaron la realización de las actividades que se especificaron en el cronograma del proyecto. Esta estructura se puede apreciar en la tabla 1 :

Tabla 1. Organización metodológica del proyecto.

\begin{tabular}{|c|c|c|}
\hline Fases & Subfases & Momentos \\
\hline \multirow{5}{*}{ I } & \multirow{3}{*}{ Reflexión } & $\begin{array}{l}\text { Didáctica de la lengua y didáctica } \\
\text { de la lectura. }\end{array}$ \\
\hline & & $\begin{array}{l}\text { Análisis de las prácticas educativas } \\
\text { de la docente investigadora. }\end{array}$ \\
\hline & & $\begin{array}{l}\text { Metacognición y didáctica de la } \\
\text { lectura. }\end{array}$ \\
\hline & \multirow[t]{2}{*}{ Formulación } & $\begin{array}{l}\text { Planeación y diseño de la propuesta } \\
\text { didáctica para la enseñanza de la } \\
\text { lectura. }\end{array}$ \\
\hline & & $\begin{array}{l}\text { Formalización de la propuesta } \\
\text { didáctica. }\end{array}$ \\
\hline \multirow{3}{*}{ II } & \multirow{3}{*}{ Ejecución } & Diagnóstico. \\
\hline & & Intervención. \\
\hline & & Evaluación. \\
\hline III & Evaluación & $\begin{array}{l}\text { Evaluación del proceso y de los } \\
\text { resultados. }\end{array}$ \\
\hline
\end{tabular}

Así, en la Fase I se llevaron a cabo los procesos de reflexión y formulación. El primero, como se señaló, centró su atención en la indagación acerca del problema de la didáctica de la lengua con el fin de determinar en qué consiste ésta, y qué aproximaciones se han dado en relación con el proceso de enseñanza-aprendizaje de la lengua. Asimismo, la reflexión en torno a esta temática general llevó al tópico de la didáctica de la lectura, esto supuso abordar la noción misma de lectura y los planteamientos que se han dado para explicar el fenómeno, así como las propuestas que de éstas se han derivado para su enseñanza. De igual forma, se analizó la noción de metacognición y el papel que este enfoque teórico tiene en el proceso de aprendizaje en general y de la lectura en particular. Por otro lado, se adelantó un análisis de la experiencia de la docente investigadora, que llevó a cabo la coordinación de la intervención didáctica con respecto a la manera como ha asumido la lectura en su quehacer profesional.

Con base en las construcciones teóricas, producto de la reflexión, se diseñó la propuesta didáctica, la cual se sustentó para su formulación en las nociones de configuración didáctica, secuencia didáctica, unidad didáctica y planificación didáctica. Una vez planteado el diseño, la propuesta se formalizó por medio de la definición de las unidades didácticas que se establecieron con base en los procesos cognitivos: centrar la atención, analizar, organizar y elaborar. Es importante anotar que estas unidades se conformaron de acuerdo a objetivos, logros, indicadores de logro, contenidos, metodología y evaluación.

En la Fase II, se adelantó la ejecución de la propuesta didáctica. Esta intervención se realizó, en la Institución Educativa Distrital Lorencita Villegas de Santos-Obrero Unión Social, Sede A, en los cursos 401 y 502 de educación básica primaria ${ }^{6}$. Para esta aplicación, en primer lugar, se hizo un diagnóstico sobre los conocimientos metacognitivos de las estudiantes y el nivel de comprensión lectora de las mismas; para esto se usó un cuestionario metacognitivo y una prueba de comprensión lectora, las cuales se aplicaron al inicio del proceso de intervención y al finalizar el mismo. En segundo lugar, se desarrollaron las unidades didácticas que se formularon en la Fase I, aspecto que corresponde a la intervención como tal, la cual contó con dos sesiones semanales, cada una de cincuenta minutos. Para efectos del desarrollo de las unidades didácticas, se recurrió a las opciones metodológicas de explicación directa y modelado cognitivo, la práctica guiada, el trabajo cooperativo y la práctica autónoma.

En la Fase III, se realizó la evaluación tanto del diseño y formulación de la propuesta didáctica como de la intervención didáctica en sí. Dicha evaluación centró su atención en el proceso que se desarrolló en cada fase, así como en los resultados que se obtuvieron al finalizar el proyecto, esto con el fin de establecer los aciertos y las falencias en la propuesta así como en la ejecución de la misma, lo

6 Esta institución educativa presta servicio de educación básica primaria, básica secundaria y media a niñas que en su mayoría proceden de familias de estrato $1,2,3$ y 4 ; de éstos, el porcentaje mayor corresponde al estrato 3 , le siguen el 2 y el 4 , y el que presenta menor proporción es el 1 . En la Sede A, se presta servicio en las jornadas de la mañana y la tarde. La jornada de la mañana atiende los cursos de educación básica primaria y los de educación media, grados 11; mientras que en la tarde asisten los cursos de educación secundaria y media, hasta el grado décimo. La intervención se adelantó, como se ha señalado, en la Sede A,(jornada de la mañana), en los grados 401 y 502 de educación básica primaria. En el grado cuarto se llevó a cabo el proyecto con la participación de 31 niñas, cuyas edades oscilaban entre los 8 y los 12 años. En el grado quinto, el trabajo se desarrolló con la participación de 26 niñas, cuyas edades se oscilaban entre los 9 y los 12 años. 
cual permitió establecer una serie de recomendaciones pedagógicas con respecto al trabajo didáctico en lectura con un componente metacognitivo en la educación básica primaria, en términos de alcances, limitaciones y posibilidades reales.

Por otro lado, las técnicas de recolección de información que se emplearon en la ejecución del proyecto forman parte de las estrategias que se emplean fundamentalmente en las investigaciones de corte cualitativo. Cabe señalar que, como sostienen Goyette y Lessard-Hébert (1988), no hay una unidad metodológica en lo que respecta a la investigación en el campo de las ciencias humanas y sociales. No obstante, se han ido consolidando y depurando una serie de técnicas e instrumentos que posibilitan pasar "de las informaciones no significativas en el plano epistémico a los datos significativos, pertinentes en relación a una problemática de investigación" (Goyette y Lessard-Hébert, 1988, p. 168). En dichas técnicas para la recolección de datos se destacan la encuesta, con sus variantes de entrevista oral o cuestionario escrito, la observación, directa o indirecta, y el análisis documental. Son éstas las técnicas a las que básicamente se recurrió en el desarrollo del trabajo. Asociados a estas técnicas se han definido una serie de instrumentos que posibilitan la recolección de los datos. Para este trabajo, se ha recurrido al cuestionario escrito, el diario de campo, las pruebas de lectura, los talleres y la observación.

Con el fin de identificar los conocimientos metacognitivos de las estudiantes que participaron en la ejecución de la propuesta didáctica, se diseñó y aplicó un cuestionario 7 , tanto al comienzo del proyecto como al final. Éste se definió con el fin de obtener, al iniciar el proceso, información sobre la noción de lectura, la manera en que las estudiantes abordan este tipo de actividad y si utilizan o no estrategias de planificación, control, supervisión y evaluación del ejercicio; a la vez, se buscaba establecer el grado de incidencia de la propuesta en la cualificación del proceso lector de los sujetos al finalizar la intervención didáctica. Esto teniendo en cuenta que el objetivo del proyecto buscaba el diseño y la validación de una propuesta didáctica para la enseñanza-aprendizaje de la lectura con una

7 El cuestionario constituye un instrumento que permite obtener datos por medio de una serie de preguntas sistemáticamente organizadas. base metacognitiva, para lo cual se hizo necesario contar con un diagnóstico del estado de las niñas en lo que a conocimientos metacognitivos se refiere, para poder establecer el grado de incidencia de la propuesta al finalizar la intervención. Para ello se utilizó, entonces, el cuestionario metacognitivo, que se definió de igual forma para los dos grados.

El cuestionario que se empleó estaba conformado por siete grandes interrogantes, referidos a aspectos propios de la lectura y, en especial, aquellos relacionados con las nociones de metalectura y metacomprensión, de tal forma que se buscaba: a) indagar sobre la noción de lectura que poseen las estudiantes; b) obtener información sobre las actividades previas que adelantan las niñas al iniciar una lectura, es decir, se buscaba establecer si efectivamente analizan y planifican la realización de una tarea de lectura; c) tener información sobre las acciones que las estudiantes adelantan durante el desarrollo de la tarea y la manera como controlan y supervisan el proceso de comprensión; d) finalmente, indagar sobre las acciones que llevan a cabo una vez han concluido una lectura, esto es, si evalúan su trabajo y cómo pueden dar cuenta de su proceso de compresión.

Para la elaboración de este cuestionario, se utilizaron preguntas cerradas con respuestas tanto excluyentes como no excluyentes (cfr. Martínez, 2002). En el primer caso, pregunta cerrada con respuesta excluyente, las preguntas son de carácter dicotómico, de tal forma que la persona tiene dos opciones y debe elegir sólo una de ellas (por ejemplo sí o no, falso o verdadero). Para el segundo caso, pregunta cerrada con respuesta no excluyente: las preguntas permiten que la persona pueda elegir varias opciones, es decir, presentan selección múltiple; en este caso, la persona puede expresar el grado de su opinión, de ahí que se plantee que se ordenan por intensidad, es decir, las opciones se asumen como válidas, pero se organizan en una escala valorativa, según el grado de importancia que el informante le otorgue a cada opción que se le presente. Según esto, el cuestionario se construyó solo con preguntas cerradas que en unos casos implicaban respuestas excluyentes y en otros no excluyentes con ordenación por intensidad8.

8 Así, las preguntas A, B y E corresponden a preguntas con respuesta no excluyente ordenada por intensidad. 
Como se señaló, el cuestionario se aplicó antes de iniciar el proceso de intervención pedagógica, $y$, una vez finalizado dicho proceso, con el fin de contrastar el estado inicial con el estado final para así establecer el grado de incidencia de la propuesta didáctica en la concepción misma de la lectura como en la forma de realizar las tareas lectoras.

Además de este cuestionario, con el fin de tener un diagnóstico con respecto al estado de la comprensión lectora de las estudiantes, al inicio y al final de la fase correspondiente a la intervención didáctica, también se diseñó una prueba de comprensión lectora, la cual tuvo como objetivo básico establecer el grado de comprensión de textos expositivos de las estudiantes. La prueba se planteó fundamentalmente para dar cuenta de un nivel de lectura que se puede catalogar como literal (cfr. Jurado, Bustamante y Pérez, 1998), esto es, aquel que permite plantear los contenidos básicos del texto: tema, ideas principales, organización del mismo y tipo de escrito.

\section{Resultados}

Los datos que se presentan en este apartado se han obtenido básicamente en la segunda fase del proyecto, ésto es, son producto de la intervención didáctica realizada durante 2007 , la cual se llevó a cabo en los cursos 401 y 502 de la IED Lorencita Villegas de Santos-Unión Social Obrera. Esta intervención supuso un momento de diagnóstico, a partir de unas pruebas de entrada (cuestionario metacognitivo y prueba de comprensión lectora), la puesta a prueba de la propuesta didáctica, aspecto que corresponde al desarrollo de las unidades didácticas definidas para cada curso, y la aplicación de las pruebas de salida (cuestionario metacognitivo y prueba de comprensión lectora). presentarán, entonces, los resultados que se obtuvieron de la aplicación del cuestionario metacognitivo antes de iniciar la intervención didáctica como tal - prueba de entrada- y después de finalizar dicha intervención -prueba de salida-; esto se hará para cada grado, pregunta por pregunta, teniendo en cuenta cada uno de los literales que las conforman y mostrando los datos de entrada y salida, así como el contraste entre éstos.

Según lo expuesto, para efectos de establecer qué conocimientos de orden metacognitivo presentaban las estudiantes antes de iniciar el trabajo, se aplicó un cuestionario metacognitivo, conformado por siete preguntas y dividido en cuatro temáticas, relacionadas con la noción de lectura, preguntas A y B, los aspectos previos a la realización de una tarea de lectura, pregunta $\mathrm{C}$, las actividades durante la lectura, preguntas $\mathrm{D}$ y E, y las acciones después de la lectura, preguntas $\mathrm{F}$ y G.

Para efectos de analizar, en términos cuantitativos, los datos obtenidos por medio de los cuestionarios aplicados a las estudiantes, se codificaron las respuestas según el tipo de pregunta con la que se trabajó, de tal forma que a los literales de cada pregunta se les asignó una letra que los identificaba. Una vez hecho esto, se creó una matriz en la que se transcribieron las distintas respuestas, por pregunta e ítem; enseguida, se procedió al conteo de las respuestas para cada ítem y al cálculo del porcentaje o índice de frecuencia de cada una de ellas. Con base en ello, se elaboraron las distintas tablas en las que se presentan los resultados para cada pregunta y sus respectivos literales. Para el caso de las preguntas excluyentes se presentan dos opciones 1 (sí) y 2 (no); para las preguntas no excluyentes, se presenta la escala valorativa que, como se señaló, para la pregunta $\mathrm{A}$ va de 1 a 6 , para la B de 1 a 7 y para la $\mathrm{E}$ del 1 a 5; en estos casos, el número 1 representa el valor más alto de la escala mientras que el 6, el 7 y el 5 corresponden a los valores más bajos de ésta, de acuerdo con la pregunta.

Las tablas que se presentan para el análisis de los cuestionarios metacognitvios están conformadas por los literales que integran cada pregunta $y$, al frente, los índices de frecuencia para cada uno de ellos, expresados en términos porcentuales, de acuerdo con la escala valorativa propuesta para las preguntas no excluyentes, la cual corresponde al número que se encuentra debajo de la línea \% entrada o \% salida, según corresponda; para el caso de las preguntas excluyentes, se presentan en una sola tabla los porcentajes de cada literal, tanto para el cuestionario de entrada y como para el de salida.

\section{Pregunta A: cuando lees un texto, ¿cuáles de los siguientes objetivos intentas conseguir?}

Ésta es una pregunta cerrada con respuesta no excluyente ordenada por intensidad; por ello está conformada por seis literales $(a-f)$, como se puede observar en la tabla 1, y con una escala de valoración que va de 1 a 6 , en la que 1 corresponde al valor más alto, mientras que 6 , al más bajo. 


\section{Grado cuarto \\ Prueba de entrada}

Tabla 2. Prueba de entrada grado cuarto.

\begin{tabular}{|c|c|c|c|c|c|c|}
\hline \multirow{3}{*}{ Ítem } & \multicolumn{6}{|c|}{ Frecuencia } \\
\hline & \multicolumn{6}{|c|}{$\%$ Entrada } \\
\hline & 1 & 2 & 3 & 4 & 5 & 6 \\
\hline a. Entender bien frase por frase & 10 & 14 & 17 & 10 & 17 & 31 \\
\hline b. Entender cada palabra & 10 & 34 & 21 & 21 & 14 & 0 \\
\hline c. Pronunciar correctamente cada palabra & 10 & 21 & 17 & 17 & 24 & 10 \\
\hline d. Entender el texto con todos sus detalles & 14 & 10 & 24 & 24 & 21 & 7 \\
\hline e. Aprender el texto de memoria & 4 & 11 & 14 & 7 & 14 & 50 \\
\hline f. Entender lo que dice el texto de forma general & 52 & 10 & 3 & 21 & 10 & 3 \\
\hline
\end{tabular}

Al revisar el consolidado de los datos obtenidos del cuestionario metacognitivo de entrada para esta pregunta, se puede evidenciar que el ítem $f$ : Entender lo que dice el texto de forma general, presenta el mayor índice de frecuencia, dado que el 52\% de las estudiantes considera que es el objetivo que busca al realizar una lectura, de ahí que lo ubiquen en el primer puesto en la escala propuesta. Siguiendo en orden descendente, se ubica el ítem $b$ : Entender cada palabra, que, aunque no obtiene el primer puesto, se ubica en el segundo lugar de importancia, de tal forma que el $34 \%$ de las niñas considera que éste es el objetivo de una tarea lectora. En tercer lugar se ubica el literal $d$ : Entender el texto con todos sus detalles, con un $24 \%$; mientras que otro grupo, correspondiente a otro $24 \%$, le asigna a este ítem el cuarto lugar. Se ubica en el último puesto, en la es- cala de valoración establecida, el ítem e: Aprender el texto de memoria, dado que el $50 \%$ de las estudiantes plantea que no es el fin de un ejercicio lector; le sigue a éste el literal a: con un $31 \%$ que considera que no es el objetivo de un ejercicio de lectura Entender bien frase por frase.

Así pues, prima en este punto la consideración de que la finalidad de adelantar una lectura de estudio es el hecho de entender de forma general lo que el texto desarrolla, de tal forma que las estudiantes dejan ver con esta respuesta que entienden la lectura como comprensión, mientras que otras formas de entenderla, como la memorización, por ejemplo, no se tienen como el aspecto fundamental al realizar un ejercicio lector.

\section{Prueba de salida}

Tabla 3. Prueba de salida grado cuarto.

\begin{tabular}{|c|c|c|c|c|c|c|}
\hline \multirow{3}{*}{ Ítem } & \multicolumn{6}{|c|}{ Frecuencia } \\
\hline & \multicolumn{6}{|c|}{$\%$ Salida } \\
\hline & 1 & 2 & 3 & 4 & 5 & 6 \\
\hline a. Entender bien frase por frase & 17 & 20 & 23 & 13 & 7 & 20 \\
\hline b. Entender cada palabra & 13 & 27 & 3 & 23 & 27 & 7 \\
\hline c. Pronunciar correctamente cada palabra & 0 & 3 & 43 & 23 & 23 & 7 \\
\hline d. Entender el texto con todos sus detalles & 27 & 27 & 23 & 20 & 3 & 0 \\
\hline e. Aprender el texto de memoria & 0 & 7 & 7 & 10 & 20 & 57 \\
\hline f. Entender lo que dice el texto de forma general & 43 & 17 & 0 & 10 & 23 & 7 \\
\hline
\end{tabular}

Cuando se examina el consolidado de los datos obtenidos del cuestionario metacognitivo de salida para esta pregunta, se observa que el ítem $f$ : Entender lo que dice el texto de forma general, sigue presentando el mayor índice de frecuencia, dado que el $43 \%$ de las estudiantes del grado cuarto considera que éste es el objetivo que busca al realizar una lectura, de ahí que lo ubiquen en el primer 
puesto de la escala. Siguiendo este análisis en orden descendente, el ítem $d$ : Entender el texto con todos los detalles, se ubica en el segundo lugar de importancia, puesto que el $27 \%$ de las niñas considera que éste es el objetivo de una tarea lectora. En tercer lugar, se ubica el literal $a$ : Entender bien frase por frase, con un 17\%; mientras que otro grupo, correspondiente a un $13 \%$, le asigna al literal $b$ : Entender cada palabra, el cuarto lugar. Por otro lado, se ubica en el último puesto, en la escala de valoración establecida, el ítem e: Aprender el texto de memoria, pues el 57\% de las estudiantes plantea que éste no es el fin de un ejercicio lector; le sigue a éste el literal a: con un 20\% de estudiantes que considera que no es el objetivo de leer Entender bien frase por frase.

Al contrastar los datos del cuestionario de entrada con los que arrojó el de salida, se puede ver que, en principio, el ítem $f$ se mantiene como el aspecto que presenta el mayor índice de frecuencia, razón por la cual obtiene el valor más importante en la escala. No obstante, como se dijo, se presenta una reducción del $9 \%$ en el número de estudiantes que en la prueba de inicio le asignaba el primer puesto a este literal; sin embargo, se presenta un aumento del $13 \%$ de las estudiantes que consideran que Entender el texto con todos sus detalles es el objetivo cuando se va leer un texto. Por otro lado, se incrementa el número de estudiantes que en el cuestionario de entrada le asignaba poca importancia a este aspecto y, por lo tanto, le adjudicaba los puntajes más bajos.

Los datos permiten plantear que se mantiene el concepto de la lectura como comprensión sobre otras nociones como la memorización o la oralización; no obstante, es evidente el hecho de que un porcentaje de las estudiantes asume como objetivo entender de forma general lo que el texto plantea, esta noción todavía no está lo suficientemente internalizada y apropiada por parte de las estudiantes, lo cual hace que no se asuma de forma general por el grupo y que se hayan incrementado los porcentajes de otros aspectos como entender bien frase por frase o entender bien palabra por palabra, por ello estos aspectos que forman parte del proceso se toman como los más importantes, de ahí que las valoraciones altas en estos ítem aumente al final de la intervención, pues el trabajo supuso el análisis detenido de los textos para llegar a su comprensión global. Esto se puede explicar -en principio- a partir del hecho de que las estudiantes manejan, como conocimiento declarativo, que la lectura supone el hecho de la comprensión, pero no son conscientes de qué es comprender, cómo adelantar esta tarea y cómo saber si se ha comprendido o no, lo que significa que sus conocimientos metalectores y metacomprensivos son todavía escasos; aunque se tome la noción de lectura como comprensión, aún está en proceso de configuración el concepto mismo de lectura y, desde luego, el de comprensión, dado que las niñas no pueden dar cuenta de forma plena en qué consiste ésta y qué aspectos involucra.

\section{Grado quinto \\ Prueba de entrada}

Tabla 4. Prueba de entrada grado quinto.

\begin{tabular}{|c|c|c|c|c|c|c|}
\hline \multirow{3}{*}{ Ítem } & \multicolumn{6}{|c|}{ Frecuencia } \\
\hline & \multicolumn{6}{|c|}{$\%$ Entrada } \\
\hline & 1 & 2 & 3 & 4 & 5 & 6 \\
\hline a. Entender bien frase por frase & 5 & 20 & 38 & 18 & 15 & 5 \\
\hline b. Entender cada palabra & 10 & 8 & 28 & 25 & 18 & 5 \\
\hline c. Pronunciar correctamente cada palabra & 23 & 25 & 8 & 10 & 30 & 5 \\
\hline d. Entender el texto con todos sus detalles & 33 & 20 & 8 & 25 & 13 & 3 \\
\hline e. Aprender el texto de memoria & 3 & 5 & 10 & 8 & 8 & 68 \\
\hline f. Entender lo que dice el texto de forma general & 38 & 23 & 8 & 10 & 10 & 13 \\
\hline
\end{tabular}

El consolidado de los datos del cuestionario de entrada muestra que el ítem que presenta el mayor índice de frecuencia para la valoración más alta es el $f$; de tal forma que prima la opinión según la cual el objetivo que persigue el 38\% de las estudiantes del grado quinto apunta a entender lo que el texto plantea; este grupo le otorga a este literal el valor más alto de la escala, mientras que un $23 \%$, si bien no le 
asigna el primer puesto, lo ubica en el segundo lugar de la escala. Para un 33\%, el objetivo es entender el texto con todos sus detalles y un $23 \%$ considera que el objetivo tiene que ver con pronunciar bien cada palabra. En el lado opuesto de la escala, el literal e es el aspecto que obtiene el mayor índice de frecuencia para la valoración más baja de la escala propuesta, de tal forma que, como se expuso, el $68 \%$ de las estudiantes manifestó que no es el objetivo de un ejercicio lector aprender el texto de memoria. Cabe señalar que en este ítem el grado de dispersión es menor con respecto a los otros, es decir, la valoración de este aspecto en los otros niveles de la escala es baja.

Según esto, se puede plantear que predomina una noción de la lectura como comprensión, ya sea del texto en términos generales o de sus detalles, frente a concepciones como la memorización. Si bien las estudiantes consideran entonces que la finalidad de un ejercicio lector apunta hacia la comprensión del contenido del texto y no tanto a la memorización, un porcentaje de las opiniones, $23 \%$, plantea que la oralización, es decir, la pronunciación correcta de las palabras que lo conforman, es una de las finalidades de la lectura, lo cual deja entrever que junto con la concepción de la lectura como comprensión, también se entiende la lectura como traducción de los signos gráficos a signos verbales.

\section{Prueba de salida}

Tabla 5. Prueba de salida grado quinto.

\begin{tabular}{|c|c|c|c|c|c|c|}
\hline \multirow{3}{*}{ Ítem } & \multicolumn{6}{|c|}{ Frecuencia } \\
\hline & \multicolumn{6}{|c|}{$\%$ Salida } \\
\hline & 1 & 2 & 3 & 4 & 5 & 6 \\
\hline a. Entender bien frase por frase & 12 & 33 & 30 & 18 & 3 & 3 \\
\hline b. Entender cada palabra & 12 & 12 & 32 & 32 & 12 & 0 \\
\hline c. Pronunciar correctamente cada palabra & 6 & 9 & 12 & 18 & 29 & 26 \\
\hline d. Entender el texto con todos sus detalles & 15 & 35 & 9 & 15 & 12 & 15 \\
\hline e. Aprender el texto de memoria & 0 & 0 & 6 & 9 & 35 & 50 \\
\hline f. Entender lo que dice el texto de forma general & 56 & 12 & 9 & 9 & 9 & 6 \\
\hline
\end{tabular}

$\mathrm{Al}$ analizar los datos consolidados del cuestionario metacognitivo de entrada con respecto a los del cuestionario de salida, se ve que se mantiene el hecho de que el literal que presenta el mayor índice de frecuencia en cuanto al nivel de importancia más alto sigue siendo el literal $f$, el cual presenta, además, un incremento del $14 \%$ en el grupo de estudiantes que plantean, que el objetivo persiguido a la hora de leer es entender lo que dice el texto de forma general, esto es, construir una representación mental del contenido fundamental del texto. Asimismo, tanto el literal $b$ como el $d$ ocupan el segundo lugar. Para el caso del literal $b$, se presenta un aumento del $5 \%$ en el porcentaje de estudiantes que le asigna el puntaje más alto, de tal forma que se pasó del $10 \%$ al $15 \%$. Por su parte, el literal $d$ presenta una reducción del $8 \%$ en el grupo de estudiantes que le asignó el primer lugar, al mismo tiempo se dio un aumento del $21 \%$ en el grupo de estudiantes que le asigna un nivel de importancia a este aspecto, pero lo deja en el segundo lugar.

Ahora bien, en el lado opuesto de la escala, también el literal $e$ sigue manteniendo el nivel de frecuencia más alto, pero se presenta una reducción del $20 \%$; se pasó del $68 \%$ al $48 \%$, pero se incrementa en un 23\%, se pasó del $8 \%$ al $33 \%$ el número de estudiantes que, si bien no le asigna el último puesto, le atribuyen el quinto lugar en la escala, es decir, considera que este aspecto no es tan importante cuando se realiza una tarea de lectura. Por otro lado, el literal $c$, que según los datos de entrada ocupó el tercer puesto, en los datos de salida pasa al último puesto, pues se da una reducción del $16 \%$ en el grupo de estudiantes que le asignaba un nivel de importancia alto, a la vez que aumenta en un $28 \%$ el número de niñas que considera que este aspecto no es el objetivo de una lectura. 
De acuerdo con esto, se hace evidente que se da una mejora en la percepción según la cual el objetivo al realizar una lectura de estudio está orientado hacia su comprensión; asimismo, se mantiene el hecho del predominio de esta concepción sobre la memorización y la oralización, de tal forma que esta concepción que mantenía cierto nivel de importancia en el cuestionario de entrada presenta una notable reducción en el grupo que la veía como aspecto central y un aumento considerable en el grupo de estudiantes que le asignó el valor más bajo de la escala, de tal forma que también se asume que no es el fin básico de un ejercicio de lectura que busca la comprensión y el aprendizaje.

\section{Pregunta B: A continuación te presentamos una lista de aspectos que muestran ¿qué se debería hacer para leer bien?}

Ordena cada una las siguientes oraciones de mayor a menor importancia, teniendo en cuenta que 1 es el mayor y 7 el menor.

Esta pregunta corresponde a una de tipo cerrada con respuesta no excluyente, ordenada por intensidad y conformada por siete ítems $(a-g)$, como se puede observar en la tabla 6 , y con una escala de valoración que va de 1 a 7 , en la que 1 equivale al valor más alto mientras que 7 , al más bajo.

\section{Grado cuarto}

Prueba de entrada

Tabla 6. Prueba de entrada grado cuarto.

\begin{tabular}{|c|c|c|c|c|c|c|c|}
\hline \multirow{3}{*}{ Ítem } & \multicolumn{7}{|c|}{ Frecuencia } \\
\hline & \multicolumn{7}{|c|}{$\%$ Entrada } \\
\hline & 1 & 2 & 3 & 4 & 5 & 6 & 7 \\
\hline a. Relacionar las ideas de las oraciones que vas leyendo & 21 & 18 & 14 & 0 & 7 & 11 & 29 \\
\hline b. Conocer todas las palabras que aparecen en el texto & 0 & 24 & 17 & 14 & 14 & 17 & 14 \\
\hline c. Pronunciar bien todas la palabras & 10 & 7 & 21 & 14 & 21 & 10 & 17 \\
\hline d. Entender lo que dice el texto & 31 & 7 & 14 & 24 & 10 & 7 & 7 \\
\hline e. Atender bien cada palabra que se lee & 7 & 24 & 14 & 24 & 10 & 14 & 7 \\
\hline f. Entender todas las frases & 7 & 7 & 10 & 14 & 31 & 31 & 0 \\
\hline g. Conseguir una idea general del contenido del texto & 24 & 14 & 10 & 10 & 3 & 10 & 28 \\
\hline
\end{tabular}

El consolidado de los datos de entrada que se obtuvo para la pregunta $B$ permite establecer que las estudiantes plantean que un aspecto que se considera adecuado para llevar a cabo un ejercicio de lectura es entender lo que dice el texto. Por esta razón, el ítem que presenta el mayor índice de frecuencia es el $d$ : Entender el texto; y llama la atención que le siga en orden de importancia, el literal g: Conseguir una idea general del contenido del texto, la cual es el resultado, de entender el texto; ésto si se tiene en cuenta que al hablar de comprensión se está planteando, que la lectura consiste en conseguir una representación mental del contenido del texto. Está en consonancia con los datos obtenidos en la pregunta $\mathrm{A}$, en donde prima el hecho de que el objetivo básico de una tarea lectora consiste en comprender el texto. Otro aspecto que está articulado con lo anterior, y ayuda a la comprensión es el ítem a que ocupa el tercer puesto: Relacionar las ideas de las oraciones que vas leyendo, ítem a. No obstante lo anterior, los datos muestran que si bien los literales $g$ y $a$ se asumen por un grupo de estudiantes como aspectos pertinentes para leer de forma adecuada, cabe señalar que también para otro grupo de estudiantes estos aspectos no son necesarios para adelantar una buena lectura, en consecuencia los ubican en el último puesto de la escala, de tal forma que estos porcentajes son superiores con respecto a los de quienes los ubican en el primer lugar.

Por otro lado, un aspecto como el $b$, que no presenta valoración en el primer lugar de la escala, sí tiene una valoración alta del $24 \%$ de las estudiantes que le asigna el segundo puesto y una dispersión en la opinión de las estudiantes entre la valoración media y baja de este aspecto, lo que muestra que no hay claridad frente al hecho de si se deben conocer todas las palabras de un texto para adelantar la lectura. 


\section{Prueba de salida}

Tabla 7. Prueba de salida grado cuarto.

\begin{tabular}{|c|c|c|c|c|c|c|c|}
\hline \multirow{3}{*}{ Ítem } & \multicolumn{7}{|c|}{ Frecuencia } \\
\hline & \multicolumn{7}{|c|}{$\%$ Salida } \\
\hline & 1 & 2 & 3 & 4 & 5 & 6 & 7 \\
\hline a. Relacionar las ideas de las oraciones que vas leyendo & 23 & 20 & 17 & 0 & 20 & 7 & 13 \\
\hline b. Conocer todas las palabras que aparecen en el texto & 20 & 20 & 10 & 20 & 13 & 10 & 7 \\
\hline c. Pronunciar bien todas la palabras & 3 & 10 & 17 & 17 & 17 & 13 & 23 \\
\hline d. Entender lo que dice el texto & 27 & 17 & 27 & 23 & 7 & 0 & 0 \\
\hline e. Atender bien cada palabra que se lee & 7 & 7 & 10 & 10 & 27 & 20 & 20 \\
\hline f. Entender todas las frases & 3 & 10 & 7 & 13 & 10 & 37 & 20 \\
\hline g. Conseguir una idea general del contenido del texto & 17 & 17 & 13 & 17 & 7 & 13 & 17 \\
\hline
\end{tabular}

$\mathrm{Al}$ analizar el consolidado de los datos que arroja el cuestionario de salida para esta pregunta, se puede ver que el aspecto que presenta el mayor índice de frecuencia en el primer puesto es el que corresponde al literal $d$, le sigue el literal $a$, el $b$ y el $g$; éstos son los que obtienen los puntajes más altos en la escala de valoración. Los literales $a$ y $g$ son complementarios del $d$, lo cual deja ver que se ratifica el hecho de que la lectura implica, ante todo, la comprensión del texto, y que esto entraña conseguir una idea general del contenido del texto y relacionar las partes del mismo para, entonces, entender lo que el texto plantea. Mientras esto se asume como lo importante, pronunciar bien las palabras o entender todas las frases no se ven como los aspectos más importantes que se deben adelantar para hacer una lectura adecuada.

Ahora bien, al contrastar los datos consolidados que arroja el cuestionario de entrada con los que ofrece el de salida, los literales que relacionan la lectura con la comprensión son los que obtienen los mayores puntajes, de tal forma que el literal $d$ : Entender lo que dice el texto, sigue siendo el aspecto que adquiere la valoración más alta, lo cual muestra que para ese grupo de estudiantes la lectura es una actividad cognitiva que tiene como finalidad la comprensión del texto. No obstante, al igual que en el caso de la pregunta A, esta noción de la lectura como comprensión no está lo suficientemente asimilada por las niñas puesto que el porcentaje de éstas que así la entiende, no es tan alto. Esto significa que la lectura es una noción en proceso de construcción y configuración. La dispersión en las percepciones y valoraciones que se observan en la tabla, a tal punto que un aspecto como el $b$, que no tenía calificación de importante en el cuestionario de entrada, ocupa el tercer puesto en el de salida. Esto también deja ver que la noción de comprensión tampoco se ha consolidado plenamente, dado que este grupo de estudiantes plantea que para obtener la representación general del contenido del texto se deben conocer todas las palabras de éste, aspecto que no necesariamente es fundamental para comprender el texto. Nuevamente, los conocimientos de metalectura y metacomprensión no se afianzan plenamente, lo cual supone que están en proceso de construcción. Por ello, la importancia de seguir con el trabajo a largo plazo para llegar a que las estudiantes manejen plenamente dichos conocimientos y, en consecuencia, mejoren su competencia lectora.

\section{Grado quinto \\ Prueba de entrada}

Tabla 8. Prueba de entrada grado quinto.

\begin{tabular}{|c|c|c|c|c|c|c|c|}
\hline \multirow{3}{*}{ Ítem } & \multicolumn{7}{|c|}{ Frecuencia } \\
\hline & \multicolumn{7}{|c|}{$\%$ Entrada } \\
\hline & 1 & 2 & 3 & 4 & 5 & 6 & 7 \\
\hline a. Relacionar las ideas de las oraciones que vas leyendo & 18 & 30 & 8 & 13 & 5 & 8 & 20 \\
\hline b. Conocer todas las palabras que aparecen en el texto & 8 & 18 & 13 & 8 & 38 & 13 & 5 \\
\hline c. Pronunciar bien todas la palabras & 13 & 13 & 8 & 10 & 18 & 8 & 33 \\
\hline
\end{tabular}




\begin{tabular}{|c|c|c|c|c|c|c|c|}
\hline d. Entender lo que dice el texto & 18 & 18 & 25 & 23 & 3 & 15 & 0 \\
\hline e. Atender bien cada palabra que se lee & 13 & 15 & 30 & 18 & 13 & 10 & 3 \\
\hline f. Entender todas las frases & 8 & 5 & 0 & 20 & 15 & 38 & 15 \\
\hline g. Conseguir una idea general del contenido del texto & 48 & 8 & 10 & 5 & 3 & 5 & 23 \\
\hline
\end{tabular}

Al examinar el consolidado de los datos de entrada para esta pregunta, se aprecia que el elemento que presenta el mayor índice de frecuencia en lo que a la valoración más alta se refiere, es conseguir una idea general del texto, correspondiente al ítem $g$. En segundo lugar, hay coincidencia entre los ítem $a$ y $d$, de tal forma que un $18 \%$ de las estudiantes considera que para hacer una buena lectura es importante relacionar las ideas de las oraciones que se van leyendo, y para otro $18 \%$ un aspecto importante para leer de forma adecuada es entender lo que plantea el texto. Así, se puede ver que estos tres aspectos, relacionados con la comprensión y complementarios se constituyen en los que alcanzan los mayores índices de frecuencia. Mientras que los demás literales obtienen unos porcentajes bajos, se observa que la oralización o conocer todas las palabras del texto no son fundamentales para realizar una buena lectura.

Lo anterior nos explica,por una parte, que las estudiantes siguen considerando que la compren- sión es aspecto central en la lectura, lo cual está en consonancia con los datos obtenidos en la pregunta A, en la que -como se indicó- los resultados señalaban que el objetivo de una lectura está asociado, en principio, a la comprensión del mismo, entendiendo ésta como la consecución de una representación general del contenido del texto, la que se obtiene relacionando las ideas que se van identificando a medida que se avanza en la lectura. Prima, además, la noción de la globalidad frente a lo local, es decir, la comprensión apunta a lo general del texto, mientras que entender frase por frase o palabra por palabra no se ve como lo más importante; así la lectura no es un proceso que supone como lo más importante el reconocimiento de las unidades léxicas o frásticas, sino la reconstrucción del significado global del texto.

\section{Prueba de salida}

Tabla 9. Prueba de salida grado quinto.

\begin{tabular}{|c|c|c|c|c|c|c|c|}
\hline \multirow{3}{*}{ Ítem } & \multicolumn{7}{|c|}{ Frecuencia } \\
\hline & \multicolumn{7}{|c|}{$\%$ Salida } \\
\hline & 1 & 2 & 3 & 4 & 5 & 6 & 7 \\
\hline a. Relacionar las ideas de las oraciones que vas leyendo & 26 & 26 & 21 & 9 & 6 & 3 & 9 \\
\hline b. Conocer todas las palabras que aparecen en el texto & 3 & 3 & 6 & 35 & 12 & 24 & 18 \\
\hline c. Pronunciar bien todas la palabras & 6 & 3 & 6 & 12 & 21 & 24 & 29 \\
\hline d. Entender lo que dice el texto & 26 & 35 & 18 & 3 & 12 & 0 & 6 \\
\hline e. Atender bien cada palabra que se lee & 6 & 9 & 15 & 12 & 21 & 35 & 3 \\
\hline f. Entender todas las frases & 0 & 9 & 12 & 21 & 24 & 9 & 26 \\
\hline g. Conseguir una idea general del contenido del texto & 32 & 15 & 24 & 9 & 6 & 6 & 9 \\
\hline
\end{tabular}

El consolidado de los datos de salida para esta pregunta permite observar que si bien el ítem $g$ sigue teniendo un índice de frecuencia alto, el porcentaje de estudiantes que planteaba que éste era un aspecto importante al leer se reduce en un $18 \%$, mientras que el ítem a se incrementa en un 12\%, obteniendo así el mismo índice que el literal $g$. Lo anterior muestra que aunque se mantiene el hecho de que entender el texto es un aspecto central para considerar que una lectura es buena, también se establece por parte de un grupo de estudiantes que relacionar las ideas del texto es un hecho igualmente importante para garantizar una buena lectura. Entre tanto, el literal $d$ presenta un incremento del $4 \%$, de tal forma que un $22 \%$ de las estudiantes ve este aspecto como indicador significativo para un ejercicio de lectura adecuado; asimismo, se incrementa el grupo de estudiantes que le asigna a este literal $d$ el segundo 
valor de la escala al pasar del $18 \%$ al $41 \%$, con lo cual están reconociendo el papel positivo de éste en la lectura. Por otro lado, aspectos como conocer todas las palabras del texto o pronunciarlas bien, así como entender todas las frases, se asumen como hechos que no son tan importantes, de ahí que las valoraciones que obtienen sean en el rango medio y bajo de la escala, tanto así que el literal $c$ tiene el mayor índice de frecuencia en lo que al puntaje más bajo se refiere, seguido por el literal $f$.

Al comparar, entonces, los datos de entrada con los de salida en lo que respecta a la pregunta $B$, es factible plantear que se mantiene la idea de la lectura como comprensión, pero en este caso se cualifica la percepción de que para llegar a dicha comprensión se debe ir relacionando e integrando las ideas que se desarrollan con el fin de obtener esa representación general del contenido del texto. Así, se mantiene la coherencia entre las respuestas obtenidas en la pregunta $\mathrm{A}$ y las que ofrece la pregunta $B$ con las cuales se ratifica $y$ consolida la noción de la lectura como comprensión, pero no sólo como conocimiento declarativo, sino también como procedimental y condicional en la medida que se plantea no sólo que éste es el objetivo, sino que se hace necesario llevar a cabo una serie de actividades para adelantar esta acción.

\section{Pregunta C: Antes de empezar a leer un texto, ¿llevas a cabo alguna de estas actividades? Marca con una $X$ si lo haces o no lo haces}

La pregunta $\mathrm{C}$ del cuestionario metacognitivo es del tipo pregunta cerrada con respuesta excluyente, de ahí que se ofrecen las opciones sí o no para los seis literales $(a-f)$ que la conforman.

\section{Grado cuarto}

Pruebas de entrada y salida

Tabla 10. Pruebas de entrada

y salida grado cuarto.

\begin{tabular}{|l|c|c|c|c|}
\hline \multirow{2}{*}{\multicolumn{1}{|c|}{ İtem }} & \multicolumn{4}{c|}{ Frecuencia } \\
\cline { 2 - 5 } & \multicolumn{2}{|c|}{ Entrada } & \multicolumn{2}{c|}{ Salida } \\
\cline { 2 - 5 } & $\mathbf{1}$ & $\mathbf{2}$ & $\mathbf{1}$ & $\mathbf{2}$ \\
\hline a. Defines por qué vas a leer el texto & 52 & 48 & 86 & 14 \\
\hline b. Revisas el texto que vas a leer & 65 & 35 & 100 & 0 \\
\hline c. Reflexionas sobre el tema del texto & 80 & 20 & 83 & 17 \\
\hline
\end{tabular}

\begin{tabular}{|c|c|c|c|c|}
\hline d. Te pones a leer inmediatamente & 87 & 13 & 28 & 72 \\
\hline e. Imaginas sobre qué puede tratar el tema & 72 & 28 & 97 & 3 \\
\hline $\begin{array}{l}\text { f. Te haces preguntas sobre lo que sabes } \\
\text { del tema y del texto }\end{array}$ & 67 & 33 & 80 & 20 \\
\hline
\end{tabular}

Como se puede ver, los literales relacionados con las actividades asociadas a la prelectura presentan una mejora en la percepción de las estudiantes, lo cual supondría que han entendido que antes de iniciar una lectura se hace necesario establecer el porqué del trabajo, analizar la tarea, activar conocimientos previos o tenerlos en cuenta, revisar el texto para tener una primera aproximación a éste, entre otras actividades. Así, se presenta una cualificación en el caso de plantear que se hace necesario, antes de empezar a leer un texto, definir por qué se va a leer, aquí -como se indicó- hay un aumento del $34 \%$ en la percepción positiva frente a este hecho; asimismo, se incrementa en un 35\% la percepción positiva para el caso de explorar el texto para tener una primera aproximación a éste; de igual forma se da un aumento en el caso de predecir el contenido del texto y hacer preguntas sobre los conocimientos previos que se tiene sobre el tema y el texto.

Esto muestra, entonces, que se da una mejora en el conocimiento y uso de estrategias asociadas a la fase de prelectura, lo cual se ratifica con el cambio en la opinión con respecto al planteamiento del literal d, en el sentido de ponerse a leer inmediatamente, pues en la prueba de entrada el $87 \%$ de las estudiantes manifestaba que sí se ponían a leer de forma inmediata, mientras que en los datos de salida este porcentaje se reduce en un $59 \%$, de tal forma que el $72 \%$ de las estudiantes plantea al final de la intervención que para llevar a cabo un ejercicio de lectura no se pone a leer inmediatamente, sino que, por el contrario, adelanta algunas actividades previas. En este orden de ideas, los resultados se hacen más coherentes y significativos al finalizar el trabajo pues un número considerable de niñas plantean que no se pone a leer inmediatamente. Asimismo, se incrementan los porcentajes de las actividades que se pueden adelantar antes de iniciar una lectura; mientras que en los datos de entrada el número de niñas que manifestaba que se ponía a leer inmediatamente era del $87 \%$, lo cual entraba en 
contradicción con los datos de los otros aspectos que hacen referencia precisamente a actividades previas a iniciar una lectura.

\section{Grado quinto}

\section{Pruebas de entrada y de salida}

Tabla 11. Pruebas de entrada y salida

\begin{tabular}{|l|c|c|c|c|}
\hline \multirow{2}{*}{\multicolumn{1}{|c|}{ İtem }} & \multicolumn{4}{|c|}{ Frecuencia } \\
\cline { 2 - 6 } & \multicolumn{2}{|c|}{ Entrada } & \multicolumn{2}{|c|}{ Salida } \\
\cline { 2 - 6 } a. Defines por qué vas a leer el texto & 63 & 38 & 82 & 18 \\
\hline b. Revisas el texto que vas a leer & 75 & 25 & 94 & 6 \\
\hline c. Reflexionas sobre el tema del texto & 77 & 23 & 50 & 50 \\
\hline $\begin{array}{l}\text { d. Te pones a leer inmediatamente } \\
\text { e. Imaginas sobre qué puede tratar el tema }\end{array}$ & 77 & 23 & 18 & 82 \\
\hline $\begin{array}{l}\text { f. Te haces preguntas sobre lo que sabes } \\
\text { del tema y del texto }\end{array}$ & 84 & 16 & 85 & 15 \\
\hline
\end{tabular}

Estos datos dejan ver que si bien las niñas manifiestan en el cuestionario de entrada que adelantan una serie de actividades antes de leer, se hace evidente que hay una contradicción entre las respuestas afirmativas frente al porcentaje de respuestas que plantean que no hacen nada distinto a ponerse a leer inmediatamente: el 77\% de las respuestas del literal $d$ plantea que las estudiantes se ponen a leer de forma inmediata, mientras que en los otros ítems manifiestan que adelantan otra serie de actividades previas. Esto muestra que no es claro, en el cuestionario de entrada, que efectivamente las estudiantes lleven a cabo dichas acciones de prelectura.

No obstante lo anterior, al finalizar la intervención, se puede ver que hay una mejora en los porcentajes de las preguntas que indagan sobre dichas actividades, de tal forma que las niñas han asumido que hay, y se pueden realizar, unas actividades previas para llevar a cabo una lectura, acciones que implican definir los propósitos de la tarea, explorar el texto, reflexionar y proponer hipótesis sobre el posible contenido del mismo, activar conocimientos previos. Asimismo, se hacen coherentes estas respuestas con las del ítem $d$; en este caso, el porcentaje de niñas que manifestó en un comienzo que se ponía a leer de forma inmediata se redujo del $77 \%$ al $18 \%$, lo cual evidencia un adelanto en el hecho de asumir que antes de iniciar a leer se pueden realizar una serie de actividades que tienen como fin preparar y desarrollar de mejor forma el ejercicio lector.

\section{Pregunta D: Cuando estás leyendo un texto, ¿realizas alguna de estas actividades? Marca con una $X$ si lo haces o no lo haces.}

La pregunta $\mathrm{D}$ busca identificar qué acciones ejecutan las niñas al momento de leer; esta pregunta corresponde a las del tipo cerrada con respuesta excluyente, de ahí que se ofrecen las opciones sí o no. Esta pregunta está conformada por diez ítems $(a-j)$.

\section{Grado cuarto}

Pruebas de entrada y de salida

Tabla 12. Pruebas de entrada y salida grado cuarto.

\begin{tabular}{|l|c|c|c|c|}
\hline \multirow{2}{*}{\multicolumn{1}{|c|}{ Ítem }} & \multicolumn{4}{c|}{ Frecuencia } \\
\cline { 2 - 5 } & \multicolumn{2}{|c|}{ Entrada } & \multicolumn{2}{c|}{ Salida } \\
\cline { 2 - 5 } & $\mathbf{1}$ & $\mathbf{2}$ & $\mathbf{1}$ & $\mathbf{2}$ \\
\hline $\begin{array}{l}\text { a. Te haces preguntas para saber } \\
\text { si estás entendiendo }\end{array}$ & 90 & 10 & 68 & 32 \\
\hline $\begin{array}{l}\text { b. Si no entiendes una parte, } \\
\text { te devuelves y relees }\end{array}$ & 79 & 21 & 93 & 7 \\
\hline $\begin{array}{l}\text { c. Subrayas el texto } \\
\text { d. Tomas notas de los aspectos } \\
\text { que consideras más importantes }\end{array}$ & 34 & 66 & 86 & 14 \\
\hline $\begin{array}{l}\text { e. Te das cuenta si tienes } \\
\text { dificultades en la lectura }\end{array}$ & 62 & 38 & 72 & 28 \\
\hline $\begin{array}{l}\text { f. Intentas anticipar lo que sigue } \\
\text { en el texto }\end{array}$ & 81 & 19 & 83 & 17 \\
\hline $\begin{array}{l}\text { g. Relacionas las ideas del texto } \\
\text { h. Simplemente lees y ya }\end{array}$ & 69 & 31 & 69 & 31 \\
\hline $\begin{array}{l}\text { i. Tienes en cuenta lo que sabes del tema } \\
\text { j. Lees varias veces el texto }\end{array}$ & 92 & 8 & 97 & 3 \\
\hline
\end{tabular}

Así pues, de acuerdo con estos datos, se puede plantear que se da una mejora en los resultados de los aspectos referidos a la actividades que las estudiantes realizan cuando están leyendo; en este sentido, son relevantes los resultados para aspectos como releer, subrayar, tomar notas, leer más de una vez el texto; actividades que se presentan en el cuestionario de entrada, pero que se incrementa 
el número de niñas que al finalizar la intervención plantean que sílas asumen y las realizan cuando leen. De igual forma se presenta un cambio significativo en la percepción frente a lo planteado en el literal $h$ : Simplemente leer y ya, dado que en este punto se reduce el número de estudiantes que responde de forma afirmativa y se incrementa el número de respuestas negativas al final de la intervención; hecho éste que, desde luego, está en consonancia con los resultados que se presentan en los otros ítem en los que, como se dijo, se hace referencia a acciones que se ejecutan mientras se adelanta un ejercicio lector y que ayudan a supervisar y controlar el proceso de comprensión del texto.

\section{Grado quinto}

\section{Pruebas de entrada y de salida}

Tabla 13. Pruebas de entrada y

salida grado quinto.

\begin{tabular}{|l|c|c|c|c|}
\hline \multirow{2}{*}{\multicolumn{1}{|c|}{ Ítem }} & \multicolumn{4}{c|}{ Frecuencia } \\
\cline { 2 - 5 } & \multicolumn{2}{|c|}{ Entrada } & \multicolumn{2}{c|}{ Salida } \\
\cline { 2 - 6 } & $\mathbf{1}$ & $\mathbf{2}$ & $\mathbf{1}$ & $\mathbf{2}$ \\
\hline $\begin{array}{l}\text { a. Te haces preguntas para saber } \\
\text { si estás entendiendo }\end{array}$ & 70 & 30 & 74 & 26 \\
\hline $\begin{array}{l}\text { b. Si no entiendes una parte, } \\
\text { te devuelves y relees }\end{array}$ & 92 & 8 & 97 & 3 \\
\hline $\begin{array}{l}\text { c. Subrayas el texto } \\
\text { d. Tomas notas de los aspectos } \\
\text { que consideras más importantes }\end{array}$ & 21 & 79 & 74 & 26 \\
\hline $\begin{array}{l}\text { e. Te das cuenta si tienes } \\
\text { dificultades en la lectura }\end{array}$ & 58 & 42 & 62 & 38 \\
\hline $\begin{array}{l}\text { f. Intentas anticipar lo que } \\
\text { sigue en el texto }\end{array}$ & 81 & 19 & 56 & 44 \\
\hline g. Relacionas las ideas del texto & 46 & 54 & 82 & 18 \\
\hline h. Simplemente lees y ya & 76 & 24 & 89 & 11 \\
\hline $\begin{array}{l}\text { i. Tienes en cuenta lo que sabes del tema } \\
\text { j. Lees varias veces el texto }\end{array}$ & 97 & 86 & 9 & 91 \\
\hline
\end{tabular}

Estos datos permiten ver que se presentan algunas mejoras en los resultados al comparar los datos de entrada con los de salida. En principio, según los datos del cuestionario inicial, las estudiantes manifiestan que efectivamente utilizan estrategias para controlar el proceso de lectura y comprensión $\mathrm{y}$, al finalizar, se incrementa levemente el número de estudiantes que utilizan estrategias de control, como hacerse preguntas, releer, subrayar, tomar notas, hacer predicciones, relacionar las ideas del texto. Ahora bien, el aspecto más significativo tiene que ver con la estrategia del subrayado, que presenta un incremento del 53\% en el número de niñas que utilizan este recurso como elemento que les ayuda a la comprensión del texto, esto asociado a la identificación de ideas o planteamientos centrales del texto, las cuales se pueden destacar por medio del subrayado.

Por otro lado, con respecto al literal $e$, se presenta reducción en el porcentaje de estudiantes que se da cuenta de las dificultades que tiene cuando está leyendo, aspecto clave para llevar de buena forma el control del proceso de comprensión, lo que deja ver que aún no se ha logrado una incorporación plena y consciente de las estrategias que posibiliten un mejor desempeño lector de forma autónoma y autorregulada. Asimismo, si bien las estudiantes manifiestan en el cuestionario de entrada que adelantan actividades que les permiten controlar y supervisar su proceso de lectura, asalta la inquietud sobre hasta qué punto son realmente conscientes y pueden dar cuenta del porqué de la importancia de estas actividades y qué papel desempeñan en un ejercicio de lectura. Así como también cabe preguntarse, si las respuestas no son simplemente producto de un conocimiento declarativo y memorístico, sino de un conocimiento procedimental y condicional. Sin embargo, como muestran los datos de salida, se puede ver que hay mejoras en las respuestas, lo que posibilita plantear que en principio se ha logrado consolidar el hecho de que la lectura es un proceso y que se hace necesario recurrir a una serie de acciones, estrategias si se quiere, que permiten controlar y supervisar si se está comprendiendo o no el texto y, en consecuencia, tomar los correctivos necesarios para garantizar un ejercicio de lectura adecuado.

\section{Pregunta E: Si tienes dificultades al leer el texto, ¿haces alguna de las siguientes acciones? Ordena cada uno los enunciados de mayor a menor importancia, teniendo en cuenta que 1 es el mayor y 5 el menor}

La pregunta $\mathrm{E}$, al igual que la $\mathrm{A}$ y la $\mathrm{B}$, es una pregunta cerrada con respuesta no excluyente ordenada por intensidad, conformada por cinco ítems $(a-e)$, con una escala de valoración que va de 1 a 5 , en la que 1 equivale al valor más alto mientras que 5 , al más bajo. 


\section{Grado cuarto}

\section{Prueba de entrada}

Tabla 13. Prueba de entrada grado cuarto.

\begin{tabular}{|l|c|c|c|c|c|}
\hline \multirow{2}{*}{\multicolumn{1}{|c|}{ Ítem }} & \multicolumn{5}{c|}{ Frecuencia } \\
\cline { 2 - 7 } & \multicolumn{5}{|c|}{$\%$ Entrada } \\
\cline { 2 - 7 } & $\mathbf{1}$ & $\mathbf{2}$ & $\mathbf{3}$ & $\mathbf{4}$ & $\mathbf{5}$ \\
\hline $\begin{array}{l}\text { a. Sigues leyendo sin tener en cuenta } \\
\text { la parte que no entiendes }\end{array}$ & 28 & 4 & 0 & 16 & 52 \\
\hline $\begin{array}{l}\text { b. Consultas en otros libros sobre el tema } \\
\text { c. Buscas las palabras desconocidas } \\
\text { en el diccionario }\end{array}$ & 28 & 20 & 24 & 24 & 0 \\
\hline $\begin{array}{l}\text { d. Vuelves a leer la parte del texto } \\
\text { que no entiendes, una o varias veces }\end{array}$ & 21 & 38 & 29 & 4 & 8 \\
\hline \begin{tabular}{l} 
e. Le pides ayuda a alguien \\
\hline
\end{tabular} & 13 & 25 & 29 & 33 & 0 \\
\hline
\end{tabular}

El consolidado de los datos que presenta el cuestionario de entrada para esta pregunta deja ver que la opinión de las estudiantes señala que realizan algunas acciones para solucionar los problemas que se les presentan cuando están leyendo. Entre dichas acciones, se encuentra consultar otros textos, utilizar el diccionario; en menor medida, releer y buscar ayuda. Frente a esto, el ítem que tiene el mayor índice de frecuencia baja en la escala propuesta corresponde al que plantea que si hay alguna dificultad se sigue leyendo sin hacer nada al respecto, lo cual implica cierta cualificación en este aspecto, en la medida en que las estudiantes asumen que si tienen dificultades de comprensión al leer pueden adelantar una serie de actividades que les permitan solucionarlas y adelantar de mejor manera la tarea lectora.

\section{Prueba de salida}

Tabla 14. Prueba de salida grado cuarto.

\begin{tabular}{|l|c|c|c|c|c|}
\hline \multirow{2}{*}{\multicolumn{1}{|c|}{ Ítem }} & \multicolumn{5}{c|}{ Frecuencia } \\
\cline { 2 - 7 } & \multicolumn{5}{|c|}{ \% Entrada } \\
\cline { 2 - 7 } & $\mathbf{1}$ & $\mathbf{2}$ & $\mathbf{3}$ & $\mathbf{4}$ & $\mathbf{5}$ \\
\hline $\begin{array}{l}\text { a. Sigues leyendo sin tener en cuenta } \\
\text { a parte que no entiendes }\end{array}$ & 17 & 3 & 3 & 7 & 69 \\
\hline b. Consultas en otros libros sobre el tema & 34 & 24 & 17 & 17 & 7 \\
\hline $\begin{array}{l}\text { c. Buscas las palabras desconocidas } \\
\text { en el diccionario }\end{array}$ & 14 & 34 & 38 & 14 & 0 \\
\hline $\begin{array}{l}\text { d. Vuelves a leer la parte del texto } \\
\text { que no entiendes, una o varias veces }\end{array}$ & 28 & 21 & 17 & 31 & 3 \\
\hline e. Le pides ayuda a alguien & 7 & 17 & 24 & 31 & 21 \\
\hline
\end{tabular}

Al revisar el consolidado de los datos de salida, se puede plantear que la tendencia en el caso de la pregunta E señala que se presenta mejora en el porcentaje de las estudiantes que asumen, como acción para enfrentar los problemas de comprensión, consultar otros libros o releer la parte que presenta dificultad, en consecuencia, estos literales obtienen los mayores índices de frecuencia en lo que respecta a los puntajes más altos de la escala propuesta. Según los datos, estas estrategias se constituyen en las que las estudiantes plantean que llevan a cabo para solucionar sus problemas de comprensión durante un ejercicio lector. Entre tanto, buscar las palabras desconocidas en el diccionario o buscar ayuda de alguien presentan reducción en los porcentajes de estudiantes que las ven como acciones viables para enfrentar las dificultades. De igual forma, se presenta una reducción en el porcentaje de las niñas que planteaban en el cuestionario de entrada que frente a una problema de comprensión no hacían nada especial y seguían leyendo, a la vez que se incrementa en un $17 \%$, como se expuso líneas atrás, el grupo de estudiantes que considera que seguir leyendo así no se haya entendido no es una acción válida. Esto deja ver que se da una mejora en entender y asumir que si se detecta una dificultad al leer un texto es bueno buscar soluciones para superar dicha problemática, y que entre estas opciones se encuentra consultar en otros textos sobre la temática que desarrolla el texto o releer varias veces el pasaje para comprenderlo, en todo caso no es pertinente seguir leyendo si no se ha entendido una parte del texto, porque ello puede afectar la comprensión del mismo.

\section{Grado quinto}

\section{Prueba de entrada}

Tabla 15. Prueba de entrada grado quinto.

\begin{tabular}{|l|c|c|c|c|c|}
\hline \multirow{2}{*}{\multicolumn{1}{|c|}{ Ítem }} & \multicolumn{5}{|c|}{ Frecuencia } \\
\cline { 2 - 7 } & \multicolumn{5}{|c|}{ \% Entrada } \\
\cline { 2 - 7 } & $\mathbf{1}$ & $\mathbf{2}$ & $\mathbf{3}$ & $\mathbf{4}$ & $\mathbf{5}$ \\
\hline $\begin{array}{l}\text { a. Sigues leyendo sin tener en cuenta } \\
\text { la parte que no entiendes }\end{array}$ & 11 & 8 & 3 & 17 & 61 \\
\hline b. Consultas en otros libros sobre el tema & 25 & 17 & 22 & 17 & 19 \\
\hline $\begin{array}{l}\text { c. Buscas las palabras desconocidas } \\
\text { en el diccionario }\end{array}$ & 22 & 50 & 19 & 8 & 0 \\
\hline $\begin{array}{l}\text { d. Vuelves a leer la parte del texto } \\
\text { que no entiendes, una o varias veces }\end{array}$ & 44 & 17 & 19 & 14 & 6 \\
\hline \begin{tabular}{l} 
e. Le pides ayuda a alguien \\
\hline
\end{tabular} & 6 & 8 & 31 & 39 & 17 \\
\hline
\end{tabular}


De acuerdo con el consolidado de los datos que ofrece el cuestionario de entrada, se hace evidente que el ítem que presenta el mayor índice de frecuencia corresponde al $d$, de tal forma que el $44 \%$ de las estudiantes señala que releer es una estrategia adecuada cuando no se entiende una parte del texto; asimismo, consultar en otros textos también se ve como una estrategia pertinente, al igual que consultar en el diccionario las palabras desconocidas. Ahora bien, en el nivel bajo de la escala de valoración de esta pregunta, el ítem $a$ es el que presenta el mayor índice de frecuencia, es decir, seguir leyendo pese a no entender una parte no es una acción adecuada.

Según estos datos, las estudiantes vuelven a manifestar que adelantan acciones cuando están leyendo con el fin de verificar si están comprendiendo el texto y de superar las dificultades que al respecto puedan tener, de ahí que el ítem con el índice de frecuencia negativo más alto es precisamente seguir leyendo sin tener en cuenta la parte del texto que haya presentado dificultades de comprensión.

\section{Prueba de salida}

Tabla 16. Prueba de salida grado quinto.

\begin{tabular}{|l|c|c|c|c|c|}
\hline \multirow{2}{*}{\multicolumn{1}{|c|}{ Ítem }} & \multicolumn{5}{c|}{ Frecuencia } \\
\cline { 2 - 7 } & \multicolumn{5}{|c|}{$\%$ Entrada } \\
\cline { 2 - 7 } & $\mathbf{1}$ & $\mathbf{2}$ & $\mathbf{3}$ & $\mathbf{4}$ & $\mathbf{5}$ \\
\hline $\begin{array}{l}\text { a. Sigues leyendo sin tener en cuenta } \\
\text { la parte que no entiendes }\end{array}$ & 12 & 3 & 9 & 9 & 68 \\
\hline b. Consultas en otros libros sobre el tema & 21 & 24 & 6 & 38 & 12 \\
\hline $\begin{array}{l}\text { c. Buscas las palabras desconocidas } \\
\text { en el diccionario }\end{array}$ & 9 & 38 & 32 & 9 & 12 \\
\hline $\begin{array}{l}\text { d. Vuelves a leer la parte del texto } \\
\text { que no entiendes, una o varias veces }\end{array}$ & 53 & 12 & 24 & 9 & 3 \\
\hline \begin{tabular}{l} 
e. Le pides ayuda a alguien \\
\hline
\end{tabular} & 9 & 21 & 30 & 27 & 12 \\
\hline
\end{tabular}

El consolidado de los datos de salida, frente a los de entrada, deja ver que el ítem $d$, relacionado con la relectura de los apartados que presentan dificultades de lectura, sigue siendo el aspecto que obtiene el mayor índice de frecuencia, con un incremento del 9\%, estos datos muestran, entonces, que este porcentaje de estudiantes considera que esta estrategia es adecuada para superar los problemas de comprensión. De igual forma, el literal $a$ se ve como un aspecto que no se debe adelantar y también presenta un aumento del $7 \%$ en el número de estudiantes que le asigna el puntaje más bajo de la escala. Según estos datos, releer se constituye en la estrategia básica y, en segundo lugar, consultar otros textos para indagar más sobre el tema, pero buscar ayuda de alguien no se asume como un aspecto tan importante para la comprensión de un texto que presente dificultad.

Se mantiene y consolida el hecho de que mientras se va desarrollando una tarea de lectura se pueden y deben adelantar acciones que ayuden a la comprensión del texto, de las cuales la más importante para las estudiantes es: la relectura. Con menor nivel de importancia, pero que se pueden adelantar, están consultar en otros textos sobre el tema, buscar palabras desconocidas en el diccionario y pedir ayuda a alguien, como última opción.

\section{Pregunta F: Cuando has leído el texto, consideras que lo has entendido porque (señala con una $X$ las opciones que consideres adecuadas)}

Esta pregunta tiene que ver con aspectos relacionados con el hecho de que las estudiantes puedan dar cuenta de si han comprendido o no un texto. La pregunta se ubica en el grupo de las cerradas con respuesta excluyente y está compuesta por siete ítems $(a-g)$.

\section{Grado cuarto}

\section{Pruebas de entrada y de salida}

Tabla 17. Pruebas de entrada y salida grado cuarto.

\begin{tabular}{|l|c|c|c|c|}
\hline \multirow{2}{*}{ Ítem } & \multicolumn{4}{|c|}{ Frecuencia } \\
\cline { 2 - 6 } & \multicolumn{1}{|c|}{ Entrada } & \multicolumn{2}{c|}{ Salida } \\
\cline { 2 - 6 } & $\mathbf{1}$ & $\mathbf{2}$ & $\mathbf{1}$ & $\mathbf{2}$ \\
\hline a. Puedes repetir de memoria el texto & 56 & 44 & 34 & 66 \\
\hline b. Puedes relacionar las partes del texto & 88 & 12 & 90 & 10 \\
\hline $\begin{array}{l}\text { c. No puedes repetir de memoria el texto, } \\
\text { pero sí puedes decir con tus palabras cuáles } \\
\text { son las ideas importantes del texto }\end{array}$ & 92 & 8 & 93 & 7 \\
\hline $\begin{array}{l}\text { d. No puedes sacar por ti misma alguna } \\
\text { conclusión nueva sobre el tema }\end{array}$ & 63 & 38 & 59 & 41 \\
\hline $\begin{array}{l}\text { e. Encuentras por tu cuenta ejemplos } \\
\text { para explicar una idea }\end{array}$ & 75 & 25 & 90 & 10 \\
\hline $\begin{array}{l}\text { f. Has aprendido nuevas cosas } \\
\text { g. No puedes encontrar por ti misma } \\
\text { ejemplos para explicar una idea }\end{array}$ & 96 & 4 & 97 & 3 \\
\hline
\end{tabular}


Teniendo en cuenta lo anterior, se puede ver que en los literales $b, c$ y $f$ presentan unas mejoras leves en las respuestas y que siguen manteniendo unos porcentajes altos en lo que a las respuestas positivas se refiere, lo que muestra que las estudiantes consideran que estos aspectos les brindan la posibilidad de plantear que han entendido el texto; asimismo, el ítem $e$ presenta un incremento relativamente alto como aspecto que sirve de indicador de la comprensión del texto. De acuerdo con esto, las estudiantes plantean que cuando pueden relacionar las partes del texto, parafrasear los contenidos más importantes de éste han aprendido cosas nuevas; o bien encontrar por su propia cuenta ejemplos para explicar alguna idea son opciones de las que disponen para poder dar cuenta de su ejercicio lector.

Frente a esto, también se hace significativa la reducción que se da en el literal $a$, del $18 \%$ como se indicó en su momento, en las respuestas que valoran como positivo el hecho de que repetir de memoria el texto es un indicador adecuado de la comprensión del mismo.
Por otro lado, si bien se presenta una ligera reducción en las repuestas positivas con respecto a los literales $d$ y $g$, los porcentajes siguen siendo altos, lo que lleva a plantear que posiblemente las estudiantes no entendieron el enunciado propuesto, lo que les llevó a entrar en contradicción, pues plantean que encontrar ejemplos por su propia cuenta es un aspecto que sirve para saber si se ha comprendido, pero, a la vez, no encontrar ejemplos para explicar una idea también es un aspecto que permite dar cuenta de la comprensión del texto. Lo mismo sucede con el hecho de valorar como positivo aprender nuevas cosas y no poder sacar una conclusión, lo que deja la inquietud de hasta qué punto este grupo de estudiantes efectivamente son conscientes de las acciones que permiten dar cuenta del proceso de comprensión y en qué consisten.

\section{Grado quinto \\ Pruebas de entrada y de salida}

Tabla 18. Pruebas de entrada y salida grado quinto.

\begin{tabular}{|c|c|c|c|c|}
\hline \multirow{3}{*}{ Ítem } & \multicolumn{4}{|c|}{ Frecuencia } \\
\hline & \multicolumn{2}{|c|}{ Entrada } & \multicolumn{2}{|c|}{ Salida } \\
\hline & 1 & 2 & 1 & 2 \\
\hline a. Puedes repetir de memoria el texto & 26 & 74 & 12 & 88 \\
\hline b. Puedes relacionar las partes del texto & 78 & 22 & 79 & 21 \\
\hline c. No puedes repetir de memoria el texto, pero sí puedes decir con tus palabras, cuáles son las ideas importantes del texto & 92 & 8 & 97 & 3 \\
\hline d. No puedes sacar por ti misma alguna conclusión nueva sobre el tema & 58 & 42 & 65 & 35 \\
\hline e. Encuentras por tu cuenta ejemplos para explicar una idea & 78 & 22 & 76 & 24 \\
\hline f. Has aprendido nuevas cosas & 94 & 6 & 100 & 0 \\
\hline g. No puedes encontrar por ti misma ejemplos para explicar una idea & 54 & 46 & 62 & 38 \\
\hline
\end{tabular}

Con base en estos datos, es posible plantear que se presenta cualificación en los aspectos que se pueden adelantar para dar cuenta del grado de comprensión de un texto, una vez finalizada la tarea. Al respecto, prima el hecho de que para el total de las estudiantes aprender nuevas cosas es un indicador de comprensión, al igual que poder hacer una paráfrasis del contenido del texto o relacionar sus partes. Tal y como se ha visto en los resultados de los ítems de otras preguntas, las estudiantes no asumen que poder repetir de memoria el texto sea un índice de su comprensión.

Si bien poder esbozar cuáles son los planteamientos centrales del texto es un aspecto que se valora como indicador de la comprensión del texto, cabe señalar que frente a esto se erige como elementos, en cierto modo contradictorios, que un porcentaje de las estudiantes planteen que no poder sacar por sí mismas alguna conclusión nueva sobre el tema del texto es un indicador de comprensión, o que no puedan encontrar ejemplo para explicar alguna idea. Aspectos que se hacen contradictorios en la medida en que, si se plantea que aprender cosas nuevas y poder verbalizar los aspectos centrales del texto son indicadores de comprensión, no poder plantear una conclusión sobre el texto o no encontrar ejemplos para explicar una idea no se deberían asumir como indicadores de compresión. En las posibles causas 
que hayan generado esta contradicción está el que las estudiantes no entendieron de forma adecuada la pregunta.

\section{Pregunta G: Cuando has terminado de leer el texto, ¿consideras que lo has leído bien? ¿Por qué? (marca con una $X$ los aspectos que creas adecuados)}

Esta pregunta, relacionada con las actividades de poslectura, está integrada por ocho literales $(a-h)$ y también es del tipo cerrada con respuesta excluyente.

\section{Grado cuarto}

Pruebas de entrada y de salida

Tabla 19. Pruebas de entrada

y salida grado cuarto.

\begin{tabular}{|l|c|c|c|c|}
\hline \multirow{2}{*}{\multicolumn{1}{|c|}{ İtem }} & \multicolumn{4}{|c|}{ Frecuencia } \\
\cline { 2 - 6 } & \multicolumn{2}{|c|}{ Entrada } & \multicolumn{2}{c|}{ Salida } \\
\cline { 2 - 6 } & $\mathbf{1}$ & $\mathbf{2}$ & $\mathbf{1}$ & $\mathbf{2}$ \\
\hline a. Conoces todas las palabras del texto & 56 & 44 & 43 & 57 \\
\hline b. Entendiste bien cada palabra & 78 & 22 & 90 & 10 \\
\hline c. Has entendido lo que quería decir el texto & 92 & 8 & 100 & 0 \\
\hline $\begin{array}{l}\text { d. Puedes repetir de memoria } \\
\text { lo más importante del texto }\end{array}$ & 79 & 21 & 66 & 34 \\
\hline $\begin{array}{l}\text { e. Has identificado las ideas } \\
\text { más importantes del texto }\end{array}$ & 74 & 26 & 93 & 7 \\
\hline f. No haces nada & 57 & 43 & 54 & 46 \\
\hline $\begin{array}{l}\text { g. Puedes hacer un resumen del texto } \\
\text { h. Puedes hacer un esquema que represente } \\
\text { los aspectos más importantes del texto }\end{array}$ & 96 & 4 & 89 & 11 \\
\hline
\end{tabular}

Según estos datos, se puede establecer que la noción de la lectura como comprensión se hace nuevamente manifiesta en las respuestas de las estudiantes, pues el 100\% considera que ha leído de forma adecuada porque ha entendido lo que el texto plantea y, a la vez, ha identificado las ideas principales; no obstante, se presenta una contradicción, dado que un $66 \%$ manifiesta que ha leído bien si puede repetir de memoria los aspectos más importantes del texto, lo que deja ver, como en otras preguntas, que la noción de lectura como comprensión no está definitivamente apropiada por las estudiantes y que este es un aspecto que todavía se debe afianzar. De igual forma, se mantiene la contradicción, que se manifiesta en el cuestionario de entrada, entre el grupo de niñas que dice que no hace nada después de leer y, sin embargo, en las otras preguntas responden que sí hace cosas después de concluir un ejercicio lector. Por otro lado, los literales $c, e, f y g$, que tienen que ver con cómo saber si se ha hecho un buen ejercicio lector, presentan mejoras en los porcentajes de las respuestas positivas, lo que permite plantear que las niñas identifican cómo pueden dar cuenta de un ejercicio lector.

\section{Grado quinto}

\section{Pruebas de entrada y de salida}

Tabla 20: Pruebas de entrada

y salida grado quinto.

\begin{tabular}{|l|c|c|c|c|}
\hline \multirow{2}{*}{\multicolumn{1}{|c|}{ İtem }} & \multicolumn{4}{|c|}{ Frecuencia } \\
\cline { 2 - 5 } & \multicolumn{2}{|c|}{ Entrada } & \multicolumn{2}{|c|}{ Salida } \\
\cline { 2 - 6 } & $\mathbf{1}$ & $\mathbf{2}$ & $\mathbf{1}$ & $\mathbf{2}$ \\
\hline a. Conoces todas las palabras del texto & 31 & 69 & 26 & 74 \\
\hline b. Entendiste bien cada palabra & 61 & 39 & 62 & 38 \\
\hline $\begin{array}{l}\text { c. Has entendido lo que quería } \\
\text { decir el texto }\end{array}$ & 82 & 12 & 94 & 6 \\
\hline $\begin{array}{l}\text { d. Puedes repetir de memoria } \\
\text { lo más importante del texto }\end{array}$ & 59 & 41 & 56 & 44 \\
\hline $\begin{array}{l}\text { e. Has identificado las ideas } \\
\text { más importantes del texto }\end{array}$ & 90 & 10 & 91 & 9 \\
\hline $\begin{array}{l}\text { f. No haces nada } \\
\text { g. Puedes hacer un resumen del texto }\end{array}$ & 93 & 52 & 22 & 78 \\
\hline $\begin{array}{l}\text { h. Puedes hacer un esquema que represente } \\
\text { los aspectos más importantes del texto }\end{array}$ & 74 & 26 & 91 & 9 \\
\hline
\end{tabular}

Al revisar los datos para el literal $a$, se puede ver que priman las respuestas negativas y que al finalizar la intervención hay un aumento en el porcentaje de éstas, que pasa del 69\% al 74\%.

En cuanto al literal $b$, se presenta un incremento del $1 \%$ en las respuestas positivas frente a la pregunta formulada. Ahora bien, lo que los datos muestran es que el $62 \%$ de las estudiantes considera que haber entendido bien cada palabra es un hecho positivo para decir que efectivamente ha comprendido el texto que ha leído; aspecto que entra en contradicción con los resultados del literal $a$, dado que el $74 \%$ de las estudiantes manifiesta que conocer todas las palabras del texto no significa que éste se haya entendido.

Para el caso del literal $c$, se mantiene el hecho de que obtiene el mayor número de respuestas la opción sí y que, además, se da un incremento en dicho porcentaje: se pasa del $82 \%$ al $94 \%$. Según 
esto, dicho porcentaje de estudiantes considera que ha comprendido el texto si puede plantear de qué trata y qué aspectos son los más relevantes. Esto también entra en contradicción con las respuestas de los literales $a$ y $b$, pues en estos casos se le da importancia de la comprensión local y se entrevé una concepción ascendente de la lectura, mientras que en este literal se aboga por la comprensión global, es decir, que comprender un texto implica elaborar una idea general de lo que trata; asimismo, se infiere una noción de lectura descendente.

Con respecto al ítem $d$, hay primacía de las respuestas positivas frente a las negativas $y$, a la vez, se da un leve descenso en las primeras al pasar del 59\% al 56\%; no obstante, se mantiene el hecho de considerar que es positivo poder repetir de memoria los aspectos más importantes del texto, como forma de dar cuenta de su comprensión. Esto deja ver que, si bien hay una reducción, se mantiene una concepción memorística de la lectura y la comprensión que contrasta con las respuestas del ítem $c$.

En el ítem $e$, se conserva el predominio de las respuestas positivas con un incremento del 1\%; así, el $91 \%$ de las estudiantes considera que puede decir que ha comprendido el texto si ha podido identificar las ideas más importantes que éste expone y desarrolla, aspecto en consonancia con las respuestas que se dieron en el literal $c$. De acuerdo con esto, para las estudiantes, comprender un texto implica tener una idea general de lo que éste plantea y los aspectos más relevantes que propone.

En relación con el ítem $f$, se presenta un incremento en el porcentaje de las respuestas negativas, pasando del $52 \%$, en el cuestionario inicial, a un $78 \%$ en los datos de salida. Este grupo de estudiantes manifiesta que para saber si ha comprendido el texto se hace necesario adelantar una serie de acciones que permitan dar cuenta de su grado de comprensión.

En cuanto al ítem $g$, se mantiene el hecho de que las respuestas positivas se imponen sobre las negativas con un aumento del $93 \%$ al $97 \%$. Estas respuestas están acordes con las obtenidas en el literal anterior, así que una de las cosas que se puede realizar para dar cuenta de la comprensión de un texto consiste en elaborar un resumen que presenta los aspectos más importantes que éste desarrolla.

Por último, para el literal $h$ también priman las respuestas positivas frente a las negativas; asimismo, también se da un ascenso en el porcentaje de dichas respuestas, que va del $74 \%$ al $91 \%$. Al igual que en el caso anterior, las estudiantes plantean que otra de las cosas que pueden hacer para formalizar su comprensión de un texto consiste en representar el contenido de éste por medio de esquemas, sean éstos mapas conceptuales, cuadros sinópticos, mapas mentales, entre otros.

\section{Conclusiones}

El programa de intervención pedagógica para la didáctica de la lectura, basado en estrategias metacognitivas, que se desarrolló en la Institución Educativa Distrital Colegio Femenino Lorencita Villegas de Santos-Obrero Unión Social, permitió realizar un seguimiento de los procesos lectores de las estudiantes, lo cual llevó a las consideraciones que a continuación se exponen en función de los logros y metas propuestas, así como de los desarrollos alcanzados.

Los datos que se obtuvieron del cuestionario metacognitivo que se aplicó antes y después del proceso de intervención didáctica dejan ver que, en lo que respecta a la noción de lectura, se presenta una cualificación en la concepción de la lectura como comprensión, de tal forma que prima este concepto frente a los de la lectura como oralización (traducción de lo gráfico a lo oral) o como memorización, este último aspecto ligado al hecho de entender la lectura y la comprensión como producto. De acuerdo con esto, las estudiantes asumen que la finalidad de un ejercicio lector apunta, en primera instancia, a la comprensión de los contenidos que el texto vehicula. Con respecto a las niñas del grado cuarto, este conocimiento metalector todavía está en proceso de integración y asimilación a sus esquemas cognitivos, aspecto que evidencia que el factor edad es una variable que se debe tener en cuenta al momento de abordar el trabajo con estrategias metacognitivas en la educación básica, especialmente en la primaria.

No obstante lo anterior, es posible adelantar este tipo de trabajos con la formulación de configuraciones didácticas que presenten una secuencia gradual que, con el apoyo permanente del docente, lleven a los estudiantes al reconocimiento de la lectura como un proceso en el que el individuo es un sujeto activo. Se hace, de esta forma, indispensable que se asuma la metalectura y la metacomprensión como contenidos que se trabajen en la clase de lengua, con el fin que los estudiantes tengan claro qué es la lectura y cuál es la relación de ésta con la 
comprensión, de tal forma que sean así conscientes de su desempeño lector y puedan, en consecuencia, mejorarlo.

Los datos también permiten plantear que, al asumir la noción de la lectura como proceso que busca la comprensión de un texto, a esta noción le subyace, entonces, que hay un conjunto de acciones a considerar para dar cuenta de dicho proceso. Algunas de estas acciones se ejecutan antes de iniciar la lectura, algunas durante el desarrollo de ésta y otras después de finalizar el ejercicio.

Los resultados muestran que un buen porcentaje de las niñas, tanto del grado cuarto como del quinto, reconoce e incorpora a sus conocimientos procedimentales el hecho de que es importante, antes de adelantar cualquier tarea y, particularmente, una de lectura, planificar la actividad en términos de definir por qué se va a leer el texto, qué se sabe del tema, cómo está estructurado dicho texto, entre otros aspectos. Asimismo, que durante la lectura es importante reconocer el tema, los subtemas y las ideas principales, releer si no se ha entendido o buscar ayuda para solucionar los problemas de comprensión y, lo más importante, reconocer que se tienen problemas y buscar soluciones. En este último aspecto, se hace necesario fortalecer las propuestas de intervención, pues las niñas aún no son plenamente conscientes de que tienen problemas y, por tanto, de que necesitan algún tipo de ayuda. Para esto, se debe trabajar más en el desarrollo de la autorreflexión y la autointerrogación, por medio del modelado y la guía metacognitiva. Por otro lado, actividades como resumir, parafrasear, representar por medio de mapas conceptuales u otro tipo de esquemas, se constituyen en mecanismos que posibilitan la formalización de la comprensión lectora y permiten dar cuenta de ésta.

Con respecto a estas acciones, los datos obtenidos muestran que se da una cualificación en la percepción y manejo de éstas, pues hay mejoras en el conocimiento y uso de las estrategias asociadas a las fases de prelectura, durante y después de la lectura. No obstante, se debe trabajar más en los procesos de reflexión sobre el valor que tienen estos aspectos a la hora de leer y el papel que efectivamente desempeñan en el proceso lector, de tal forma que los estudiantes los integren a sus esquemas cognitivos como conocimientos de orden condicional, que les ayudarán a llevar de mejor manera y con mejores resultados sus procesos de aprendizaje por medio de la lectura.

La prueba de comprensión lectora de diagnóstico y la de salida permiten comparar y confrontar la noción de lectura que las estudiantes tienen como experiencia preliminar al proceso de intervención y de la noción posterior al programa de intervención, evidenciando un desplazamiento en la conceptualización de lo que significa leer y de los procesos que este ejercicio conlleva; aspecto acorde con los datos que se obtuvieron en los cuestionarios metacognitivos, en los que -como se indicó- se nota la consolidación de la lectura como un proceso que busca la comprensión del texto con el que se interactúa, y que en dicho proceso el lector es un sujeto activo que debe realizar una serie de actividades que le sirven de apoyo para reconstruir el significado del texto.

También se debe destacar la importancia del saber previo como parte fundamental del diálogo llevado a cabo entre el lector y el texto, y de la toma de conciencia por parte de las niñas sobre los conocimientos de diversos temas que se ponen en juego a la hora de leer. De esta manera, las estudiantes reconocieron la importancia del saber previo y de su accionar como parte de la experiencia vital y lectora con la que enfrentan la lectura.

La experiencia desarrollada con el programa de intervención hizo posible que las estudiantes evidenciaran y apropiaran el valor de la interrogación y la autointerrogación como parte del proceso lector. Si bien es un hecho positivo que las estudiantes reconozcan el valor de estos aspectos, se hace necesario fortalecer más este trabajo, pues las estudiantes dejan ver debilidades al respecto, lo cual implica que se debe hacer más énfasis en aspecto metodológicos, como el desarrollo de la autointerrogación y la discusión metacognitiva, para efectos de comprender el valor que estos aspectos tienen al momento de desarrollar una actividad de aprendizaje.

La integración del aprendizaje, entendido aquí como el desarrollo del proceso lector llevado a cabo por cada una de las estudiantes en relación con el uso de las estrategias metacognitivas, a situaciones específicas de la vida cotidiana permitió observar algún grado de apropiación por parte de las estudiantes en relación con la construcción de conocimiento sobre la lectura. Así, la aparición de la noción de relectura en las reflexiones metacognitivas de 
las niñas permite observar la integración de la interacción entre el lector y el texto como un ejercicio de varios momentos y con la construcción de una experiencia lectora que supera los propósitos inmediatistas.

El saber construido en torno a la lectura permitió a las estudiantes entender la lectura como proceso que involucra varios momentos, pasos y secuencias, configurando así la noción de lectura como proceso cognitivo. Al respecto, la integración de acciones como formular objetivos, explorar, activar conocimientos previos, analizar, organizar, identificar ideas principales, representar, entre otras, dan cuenta de un ejercicio lector gradual y en cierta medida autorreflexivo.

La constitución de un ejercicio lector basado en la integración del uso de estrategias metacognitivas y

\section{Bibliografía}

Burón, J. (1996). Enseñar a aprender: Introducción a la metacognición. Bilbao: Mensajero.

Campenhoudt, Q. (2001). Manual de investigación en ciencias sociales. México: Limusa.

Castillo, M. y Santiago, A. (2006). Consideraciones sobre la relación entre lectura, metacognición y evaluación. Folios, 23, 91-101.

De Clerk, L. y Semons, J. (1989). Estudios de los procesos metacognoscitivos de la comprensión de lectura. En Leer en la escuela: Nuevas tendencias en la enseñanza de la lectura. Madrid: Pirámide.

Ferreiro, E. (2002). Pasado y presente de los verbos leer y escribir. México: Fondo de Cultura Económica.

Gaskins, I. y Thorne, E. (1999). Cómo enseñar estrategias cognitivas en la escuela: el manual Benchmark para docentes. Barcelona: Paidós.

Goyete, G. y Lessard-Hérbert, M. (1988). La investigación-acción: Funciones, fundamentos e instrumentación. Barcelona: Laertes.

Gvirtz, S. y Palamidessi, M. (1998). El ABC del docente: currículum y enseñanza. Buenos Aires: Aique.

Jurado, F., Bustamante, G. y Pérez, M. (1998). Juguemos a interpretar: evaluación de las competencias en lectura y escritura. Bogotá: Plaza \& Janés. la aplicación a diversos campos del saber, entendido en el ejercicio de las niñas como varias materias, permite observar la generación de hábitos de estudios y el refinamiento de los procesos de aprendizaje.

Al hablar de una práctica metacognitiva y de la generación de estrategias en procesos de comprensión lectora de textos expositivos se constituye en una práctica pedagógica importante, viable $y$, además, necesaria en los procesos pedagógicos institucionales. Los elementos que aporta la metacognición pueden cualificar el ejercicio pedagógico, al aportar estrategias útiles en el aula y hacer que el docente direccione su trabajo hacia un manejo consciente por parte suya y de sus estudiantes, quienes en últimas serán los beneficiados, llegando a tener un manejo autónomo de la lectura y su ejercicio de comprensión.h

Lerner, D. (2001). Leer y escribir en la escuela: lo real, lo posible y lo necesario. México: Fondo de Cultura Económica.

López, G. y Arciniegas, E. (2004). Metacognición, lectura y construcción de conocimiento. El papel de los sujetos en el aprendizaje significativo. Cali: Universidad del Valle.

Martínez, F. (2002). El cuestionario: un instrumento para la investigación en ciencias sociales. Barcelona: Laertes.

Mayor, J., Suengas, A. y González, J. (1993). Estrategias metacognitivas: aprender a aprender $y$ aprender a pensar. Madrid: Síntesis.

Mendoza, A. (coord.) (2003). Didáctica de la lengua y la literatura para primaria. Madrid: Pearson Educación.

Monereo, C. (coord.) (2002). Estrategias de aprendizaje. Madrid: Antonio Machado Libros.

Restrepo, B. et ál. (2004). Investigación-acción educativa: una estrategia de transformación de la práctica pedagógica de los maestros. Bogotá: Aguilar.

Santiago, A., Castillo, M. y Morales, D. (2007). Estrategias y enseñanza-aprendizaje de la lectura. Folios, 26, 27-38.

Santiago, A., Castillo, M. y Vega, J. (2005). Lectura, metacognición y evaluación. Bogotá: Alejandría Libros. 